\title{
Theory of electron-phonon dynamics in insulating nanoparticles
}

\author{
Michael R. Geller ${ }^{\mathrm{a}, *}$, W.M. Dennis ${ }^{\mathrm{a}}$, Vadim A. Markel ${ }^{\mathrm{b}}$, Kelly R. Patton ${ }^{\mathrm{a}}$, \\ Daniel T. Simon ${ }^{\mathrm{c}}$, Ho-Soon Yang ${ }^{\mathrm{d}}$ \\ ${ }^{a}$ Department of Physics and Astronomy, University of Georgia, Athens, GA 30602-2451, USA \\ ${ }^{\mathrm{b}}$ Department of Electrical Engineering, Washington University, St. Louis, MO 63130-4899, USA \\ ${ }^{\mathrm{c}}$ Department of Physics, University of California, Santa Cruz, CA 95064, USA \\ ${ }^{\mathrm{d}}$ Argonne National Laboratory, Materials Science Division, 9700 S. Cass Avenue, Argonne, IL 60439, USA
}

\begin{abstract}
We discuss the rich vibrational dynamics of nanometer-scale semiconducting and insulating crystals as probed by localized electronic impurity states, with an emphasis on nanoparticles that are only weakly coupled to their environment. Two principal regimes of electron-phonon dynamics are distinguished, and a brief survey of vibrationalmode broadening mechanisms is presented. Recent work on the effects of mechanical interaction with the environment is discussed. (C) 2002 Elsevier Science B.V. All rights reserved.
\end{abstract}

Keywords: Nanoparticles; Phonons; Electron-phonon interaction

\section{Introduction}

Recently, there has been considerable interest in nanoparticles and nanocrystals because of their unique physical properties and for their potential use in nanotechnology [1]. Like quantum dots, nanocrystals made from semiconductors and insulators have a discrete electronic spectrum, but unlike quantum dots they also have a nearly discrete vibrational spectrum with a suppressed phonon density-of-states (DOS) at low energy. In particular, an isolated spherical nanoparticle of diameter $d$ cannot support internal vibrations at

\footnotetext{
*Corresponding author. Tel.: + 1-706-542-2834; fax: +1706-542-2492.

E-mail address: mgeller@physast.uga.edu (M.R. Geller).
}

frequencies less than the so-called Lamb mode frequency, about $2 \pi v_{\mathrm{t}} / d$, where $v_{\mathrm{t}}$ is the bulk transverse sound velocity [2]. Any property of a nanoparticle that depends on the low-frequency vibrational spectrum, such as its low-temperature thermodynamic properties or low-energy electronic relaxation rate, will be different than in bulk crystals. This will be especially dramatic for nanoparticles - for example, prepared in powder form-that are only weakly coupled to their environments.

One way to probe the vibrational spectrum of a nanoparticle is to optically excite an electronhole pair and study the intraband electronic energy relaxation prior to radiative recombination [3]. However, the excitonic states, being only weakly localized, will suffer significant 
quantum-confinement effects in the nanoparticle, making comparison with bulk relaxation rather indirect. An alternative probe of the vibrational spectrum

is provided by well-localized electronic impurity states in a doped nanocrystal. The impurity states can be used to probe both energy relaxation by phonon emission [4] and phonon-induced dephasing [5]. In these cases, the difference between the nanocrystal and bulk behavior is almost entirely a consequence of their differing vibrational modes.

Yang et al. [4] measured the phonon DOS of $\mathrm{Y}_{2} \mathrm{O}_{3}$ nanoparticles, in powder form, with a size distribution ranging from 7 to $23 \mathrm{~nm}$ in diameter. $\mathrm{Y}_{2} \mathrm{O}_{3}$ nanoparticles $15 \mathrm{~nm}$ in diameter cannot support phonons with energies below about $10 \mathrm{~cm}^{-1}$. The phonon DOS was obtained by measuring the nonradiative decay rate of an excited electronic state of $\mathrm{Eu}^{3+}$, and at $3 \mathrm{~cm}^{-1}$ was found to be about 100 times smaller than that in a bulk $\mathrm{Y}_{2} \mathrm{O}_{3}$ crystal.

In this paper we discuss recent theoretical work [6-9] motivated by the experiment of Yang et al. [4]. After discussing the two principal regimes of electron-phonon dynamics, a brief survey of vibrational-mode broadening mechanisms is presented, and we discuss new work on the phonon DOS in a nanoparticle mechanically coupled to its surroundings.

\section{Relaxational versus vibronic dynamics}

The simple discussion presented above suggests that phonon emission should be considerably suppressed below frequencies of the order of $2 \pi v_{\mathrm{t}} / d$. However, this cannot be the whole story, as can be seen by considering the limit of a localized electronic impurity state in a completely isolated nanoparticle. In this case energy is exchanged between the electronic and vibrational degrees of freedom in a oscillatory manner, and no relaxation would be observed. Indeed, an impurity state in an isolated nanoparticle may be regarded as a phonon analog of an atom in a cavity, which is known to exhibit Rabi-like population dynamics [10].
We are therefore led to the conclusion that there will be two distinct regimes characterizing the nature of low-energy electron-phonon dynamics in a semiconducting or insulating nanoparticle. In what we shall refer to as the relaxational regime, an excited-state electron population decays approximately exponentially in time with a rate correctly given by Fermi's golden rule. In the vibronic (or Rabi) regime, however, the population oscillates for some number of cycles before decaying. The crossover between these regimes occurs when the width of the broadened Lamb mode becomes equal to the electron-phonon interaction strength.

It is tempting to conclude that it would be straightforward to determine experimentally whether a system of nanoparticles was in the relaxational or vibronic regimes, but the effects of ensemble-averaging complicate the matter [6]. We have found that at short times the ensembleaveraged excited-state population oscillates but has a decaying envelope. At long times, however, the oscillations become purely sinusoidal about a "plateau" population, with a frequency determined by the electron-phonon interaction strength, and with an envelope that decays algebraically as $t^{-1 / 2}$. Whether one can observe luminescence from the plateau region depends on the available experimental resolution.

\section{Intrinsic phonon-broadening mechanisms}

Phonon broadening can come from mechanisms operative within a nanoparticle, or from its interaction with the environment. The former tends to be more important at high energies and the latter at low energies.

For example, anharmonic interaction, which is always present, will cause the modes to broaden, leading (at finite temperature) to a DOS at energies below $2 \pi v_{\mathrm{t}} / d$. However, anharmonicity is ineffective at low energy and a calculation of the anharmonic broadening showed that the resulting DOS is much smaller than that observed [7]. It is clear from energy conservation that a lowtemperature DOS below $2 \pi v_{t} / d$ must come 
from interaction of the nanoparticle with its surroundings.

\section{Extrinsic phonon-broadening mechanisms}

An important extrinsic broadening mechanism results from the fact that the nanoparticles of Ref. [4] are not isolated, but rather are in contact with each other or some support structure. This contact enables the nanoparticles to couple to an environment with a continuous spectrum at low energy. Preliminary work suggests that this environmental interaction broadens the vibrational modes enough to explain the observed DOS [8].

We consider a single isotropic elastic sphere of diameter $10 \mathrm{~nm}$, representing the nanoparticle, connected to a semi-infinite isotropic elastic continuum lying in the $x y$ plane and extending to infinity in the negative $z$ direction. For simplicity, we take the substrate and the nanoparticle to be made of the same material. We model the contact between the two by a weak harmonic spring, corresponding to the situation where the nanoparticle and substrate are connected by only a few atomic bonds or by a small "neck" of material. The Hamiltonian for the system is

$$
\begin{aligned}
H= & \sum_{J} \omega_{J} a_{J}^{\dagger} a_{J}+\sum_{I} \omega_{I} b_{I}^{\dagger} b_{I} \\
& +: \frac{K}{2}\left[u_{\mathrm{n}}^{z}\left(\mathbf{r}_{0}\right)-u_{\mathrm{s}}^{z}\left(\mathbf{r}_{0}\right)\right]^{2}:
\end{aligned}
$$

where the $a_{J}$ and $a_{J}^{\dagger}$ are annihilation and creation operators for phonons in the nanoparticle, and the index $J$ runs over all the modes of the nanoparticle. The $b_{I}, b_{I}^{\dagger}$, and $I$ correspond to the substrate phonons. The spring constant $K$ is taken to be of the order of an atomic bond strength of the material, and the pair of colons :: indicates normal ordering. $\mathbf{u}_{\mathrm{n}}(\mathbf{r})$ and $\mathbf{u}_{\mathrm{s}}(\mathbf{r})$ are the phonon displacement fields of the nanoparticle and the substrate, respectively, and $\mathbf{r}_{0}$ is the point of connection.

The phonon DOS is obtained by calculating the nanoparticle phonon propagator to leading order in $K$ and using the quasiparticle-pole approximation. This method is equivalent to using Fermi's golden rule to calculate phonon lifetimes and assuming Lorentzian line shapes. At $3 \mathrm{~cm}^{-1}$ the DOS is found to be $5.8 \times 10^{-6}$ states per $\mathrm{cm}^{-1}$, or about $3.2 \times 10^{-4}$ times the bulk DOS. Experimentally, the ratio of nanoparticle to bulk DOS was found to be approximately $7.4 \times 10^{-3}$, about 20 times larger than our result. This is reasonable considering the simplicity of our model. However, the quasiparticle-pole approximation is inaccurate at low energies, where the deviation from Lorentzian line shapes becomes important. But the order-of-magnitude agreement does suggest that we have correctly identified the relevant broadening mechanism in these nanoparticles. An accurate calculation of the phonon DOS, based on the solution of the Dyson equation, is in progress.

The possibility that electronic energy relaxation can be caused by fictitious forces acting in an oscillating nanoparticle has also been investigated [9], but we do not believe that this somewhat exotic mechanism is operative here. A broadening mechanism that we have not yet considered is the effect of adsorbed molecules or "dirt" on the outside of the nanoparticle, which may also dampen the vibrational modes.

\section{Acknowledgements}

This work was supported by NSF Grants DMR-0093217 and DMR-9871864, and by a Research Innovation Award and Cottrell Scholars Award from the Research Corporation. It is a pleasure to thank Steve Lewis and Richard Meltzer for useful discussions.

\section{References}

[1] For a survey of interesting nanocrystal geometries, see A.P. Alivisatos, Science 271 (1996) 933, and references therein.

[2] H. Lamb, Proc. London Math. Soc. 13 (1882) 187.

[3] U. Woggon, Optical Properties of Semiconductor Quantum Dots, Springer, Berlin, 1997;

S.V. Gaponenko, Optical Properties of Semiconductor Nanocrystals, Cambridge University Press, Cambridge, 1998. 
[4] H.S. Yang, S.P. Feofilov, D.K. Williams, J.C. Milora, B.M. Tissue, R.S. Meltzer, W.M. Dennis, Physica B 263 (1999) 476.

[5] R.S. Meltzer, K.S. Hong, Phys. Rev. B 61 (2000) 3396.

[6] D.T. Simon, M.R. Geller, Phys. Rev. B 64 (2001) 115412.

[7] V.A. Markel, M.R. Geller, J. Phys. Condens. Matter 12 (2000) 7569 .
[8] K.R. Patton, M.R. Geller, J. Lumin. 94 (2001) 747.

[9] H.S. Yang, M.R. Geller, J. Lumin. 83 (1999) 317; H.S. Yang, M.R. Geller, W.M. Dennis, Phys. Rev. B 62 (2000) 9398.

[10] M.O. Scully, M.S. Zubairy, Quantum Optics, Cambridge University Press, Cambridge, 1997. 